\title{
Évaluation monétaire des services écosystémiques. Un exemple d'usage dans la mise en place d'une politique de l'eau en France
}

\author{
Sarah Feuillette ${ }^{1}$, Harold Levrel ${ }^{2}$, Stéphanie Blanquart ${ }^{3}$, Olivier Gorin ${ }^{4}$, Guillaume Monaco ${ }^{5}$, \\ Bruno Penisson ${ }^{6}$, Stéphane Robichon ${ }^{7}$
}

1 Responsable du service Prévision, évaluation et prospective, Agence de l'eau Seine-Normandie, 92027, Nanterre, France

2 Économiste, IFREMER, UMR AMURE, 29280 Plouzané, France

3 Économiste, Agence de l'eau Loire-Bretagne, 45063 Orléans, France

4 Économiste, Agence de l'eau Rhône-Méditerranée, 69363 Lyon, France

5 Économiste, Agence de l'eau Rhin-Meuse, 57160 Rozérieulles, France

6 Économiste, Agence de l'eau Artois-Picardie, 59508 Douai, France

7 Économiste, Agence de l'eau Adour-Garonne, 31078 Toulouse, France

L'évaluation économique des services environnementaux est devenue incontournable dans les modes de justification des politiques publiques. NSS a déjà exploré dans divers contextes les origines et les implications de cette évaluation devenue prédominante (NSS, 2012, 20, 1, 3-15 ; NSS, 2013, 21, 4, 363-372). Le cas présenté ici traite des politiques de l'eau dérivées de la mise en œuvre de la directive-cadre. Cet article donne la parole à des économistes, qui ont été au cœur de son application au sein des agences de l'eau. Les auteurs mettent en évidence toutes les fragilités inhérentes à la monétarisation de bénéfices non marchands, mais aussi un cadrage strict qui n'empêche pas une diversité dans la mise en œuvre. Enfin, ils invitent à considérer plus largement les bénéfices de la restauration du bon état écologique d'une masse d'eau, considérations financières comprises, à condition qu'y figurent explicitement les enjeux politiques sous-jacents à toute procédure d'évaluation.

La Rédaction

Mots-clés :

environnement ; dispositifs institutionnels; eau ; évaluation économique ; coûts disproportionnés

\section{Keywords:}

environment; institutional systems; water; economic valuation; disproportionate costs
Résumé - L'État a sollicité les agences de l'eau françaises pour justifier des dérogations à l'atteinte du «bon état » des eaux en 2015, pour cause de coûts disproportionnés, sur un certain nombre de petits bassins versants. En accord avec le ministère chargé de l'environnement, il a été convenu de s'appuyer pour ce faire sur des analyses coûts-bénéfices. Le présent article rend compte de cette expérience et des questionnements suscités par le chiffrage des bénéfices non marchands, qui se sont posés en termes techniques, stratégiques et éthiques, pour pointer plus globalement les limites de l'usage des référentiels économiques dans le cadre de l'application d'une politique environnementale.

\begin{abstract}
Monetary valuation of ecosystem services. An example through a water policy implementation in France. In the context of the Water Framework Directive, the French Water Agencies were asked to justify exemptions, for disproportionate cost reasons, to the "good water" status in a series of river basins in 2015. The ministry of the Environment and the economists of the Water Agencies agreed on the fact that this justification would be based on cost-benefit analyses. A great number of costbenefit analyses were carried out on water bodies or groups of water bodies. This article reports on this experience conducted by the French Water Agencies. It highlights the fact that the large majority of restoration projects generated higher costs than benefits. It suggests that the implementation of the Water Framework Directive could be considerably limited by the use of the Costs-Benefits-Analysis. This result is discussed, especially with regard to the assessment of non-market benefits. These methods are in fact a source of much technical uncertainty which can be used in a strategic way to justify lack of implementation of the EU Directive. Last, the article questions the limits of economic referentials against the legal referential in the implementation of environmental policy.
\end{abstract}

Auteur correspondant : S. Feuillette, feuillette.sarah@aesn.fr 


\section{Introduction}

L'économie et le droit sont les seules sciences sociales qui proposent des référentiels normatifs sur la manière dont les politiques publiques doivent être menées (Kirat, 2012). Le référentiel normatif du droit est la règle. Celui de l'économie est le bien-être social.

Le référentiel légal vise à guider l'application de politiques publiques au regard de textes adoptées par des représentants directs ou indirects des citoyens. Le référentiel économique propose de guider les politiques publiques au regard d'indicateurs monétaires, notamment ceux reflétant l'ensemble des gains et des pertes d'utilité des individus composant une population.

Les référentiels légaux sur lesquels s'appuient les politiques publiques sectorielles sont aujourd'hui de plus en plus mis en balance avec les référentiels économiques. La prise en compte des référentiels économiques a pour objectif d'évaluer monétairement et de rationaliser la mise en œuvre des politiques publiques de la santé, du transport, de l'éducation ou de l'environnement, en mobilisant un « référentiel global» qui transcende les " référentiels sectoriels » associés aux spécificités de ces politiques (Muller, 2000). Concrètement, l'argument économique vise le plus souvent à démontrer que les bénéfices de la mise en œuvre d'une politique publique sectorielle vont être supérieurs aux coûts de cette politique.

Dans le domaine de l'environnement, l'analyse coûtsbénéfices des projets environnementaux a été largement développée depuis une quinzaine d'années (Heal, 2000 ; Pearce et Seccombe-Hett, 2000). La principale difficulté rencontrée par ces évaluations est de pouvoir mesurer les bénéfices environnementaux associés aux usages non marchands tels que les activités récréatives en rapport avec la biodiversité, ou le simple fait de bénéficier d'un paysage naturel à proximité de chez soi, ce qui peut conduire à sous-évaluer les bénéfices globaux. Plusieurs travaux scientifiques récents ont cependant permis de démontrer que les bénéfices associés à la restauration d'écosystèmes naturels étaient supérieurs aux coûts de mise en œuvre de ces projets de restauration (TEEB, 2010 ; de Groot et al., 2013), mais il s'agit de travaux scientifiques qui ne sont pas vraiment liés à des politiques publiques.

Il existe ainsi peu d'exemples concrets de réelle mobilisation des évaluations économiques dans des politiques environnementales (Laurans et al., 2013). Une exception notable de politique publique environnementale dans laquelle le recours aux évaluations monétaires a été largement sollicité est celle de la directive-cadre sur l'eau (DCE). Dans ce cadre institutionnel, des analyses coûts-bénéfices sont en effet requises pour évaluer le caractère éventuellement "disproportionné » du coût des projets qu'implique cette politique au regard des bénéfices générés et justifier ainsi des dérogations à l'atteinte du bon état des masses d'eaux à l'horizon 2015, tel que défini réglementairement.

Ce principe de soumettre le référentiel légal du bon état à celui des coûts et des bénéfices de l'atteinte de cet objectif pour la société peut apparaître comme tout à fait justifié. En effet, si le coût social d'une mesure environnementale est considéré " disproportionné », alors il doit pouvoir être possible d'en limiter la portée. C'est la raison pour laquelle cette précaution a été explicitement mentionnée dans le texte. Pour autant, l'application de ce critère fait peser le risque de réduire considérablement la portée environnementale du cadre légal. Cela explique pourquoi il est essentiel que les méthodes mobilisées pour calculer ces coûts et ces bénéfices sociaux soient suffisamment robustes.

Cet article a pour objectif de montrer les incertitudes qui pèsent sur le processus d'évaluation tel qu'il est mené dans le cadre de la DCE, d'identifier les espaces d'interprétation que ces incertitudes créent, de pointer la manière dont ces espaces peuvent être utilisés dans une perspective stratégique en vue de réduire la portée d'une politique publique environnementale et enfin de discuter les forces et les limites de l'usage des référentiels économiques dans le cadre de l'application d'une politique environnementale.

\section{Les référentiels normatifs légaux et économiques dans le cadre de la DCE}

La directive-cadre européenne sur l'eau, publiée en 2000, demande aux États membres d'atteindre le «bon état ${ }^{1}$ » des «masses d'eau » (tronçons de cours d'eau, nappes et portions de littoral) d'ici 2015. C'est la première directive européenne à autant solliciter l'analyse économique dans ses textes (Laurans, 2006). Elle traduit une tendance de fond de la mise en œuvre des politiques européennes, partiellement inspirée des orientations britanniques: une plus grande fermeté sur les objectifs et les résultats, une plus grande subsidiarité quant aux moyens à employer, et un recours à la justification économique des choix subsidiaires (De Abreu, 2001).

\footnotetext{
1 Le «bon état » est défini par la DCE selon plusieurs paramètres. Pour les cours d'eau, il dépend, d'une part, d'un «bon état écologique ", lui-même composé du « bon état biologique » (décrit par des indicateurs tels que l'indice poisson, l'indice diatomées, l'indice macrofaune qui mesurent la diversité et le nombre de familles plus ou moins visibles à l'œil nu) et du « bon état physicochimique » (décrit par la température et le $\mathrm{pH}$ de l'eau, sa teneur en oxygène, en nitrates...), d'autre part, du « bon état chimique » décrit par la présence ou non de substances telles que des pesticides, des métaux lourds...
} 


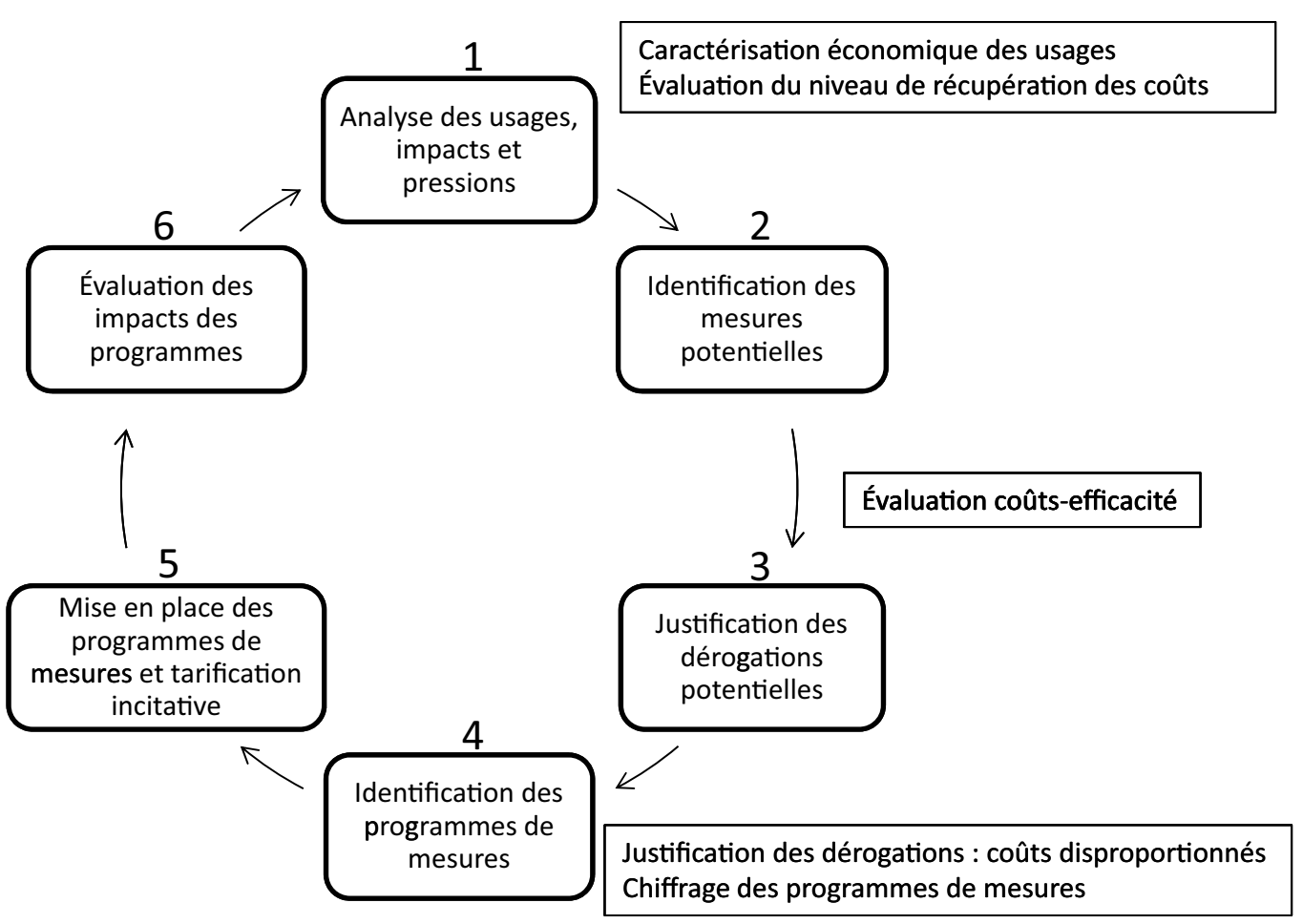

Fig. 1. Le cycle de l'analyse économique dans la directive-cadre sur l'eau (d'après Salvetti, 2013, p. 5).

L'analyse économique est mobilisée à plusieurs reprises dans la mise en œuvre de la DCE (Fig. 1).

L'analyse économique pour la DCE qui nous intéresse ici (étape 3 de la figure 1) se situe lors de la conception du "plan de gestion", incarné en France dans le SDAGE (Schéma directeur d'aménagement et de gestion des eaux), qui préexistait à la DCE. Celui-ci fixe, à l'échelle du district hydrographique, les objectifs environnementaux pour chaque masse d'eau, tandis que le «programme de mesures » doit permettre d'atteindre les objectifs environnementaux à partir d'une liste d'actions à mettre en œuvre. L'analyse économique doit permettre, à ce stade, de :

- choisir les mesures les plus efficaces au moindre coût pour atteindre l'objectif visé ;

- justifier d'éventuels reports de délai (c'est la partie qui nous intéresse ici), sachant que la DCE, qui se décline en trois cycles temporels de 6 ans, propose trois horizons possibles pour atteindre le bon état des eaux : 2015, 2021 ou 2027 ;

- justifier un objectif moindre : au lieu de viser le bon état global, on visera un « bon état partiel», ce qui revient à dire que tous les paramètres ne seront pas classés «bons" mais que certains pourront être «moyens", voire « médiocres".

Des dérogations de délais ou d'objectifs peuvent être justifiées à partir d'argumentaires de trois types : «techniques» (on ne dispose pas de la technique per- mettant d'atteindre l'objectif), «naturels » (le temps de réaction des milieux naturels ne permet pas de respecter l'échéance) ou enfin « économiques » (le coût du projet à mettre en œuvre pour atteindre l'objectif est jugé disproportionné). Le critère économique doit être mobilisé en dernier recours d'après une circulaire nationale évoquée plus loin ${ }^{2}$. Nous nous intéresserons dans la suite de cet article à cette question du coût disproportionné.

\section{Les coûts disproportionnés}

Dans son article 4, le texte de la DCE indique que des coûts disproportionnés peuvent justifier :

- la désignation d'une masse d'eau comme « fortement modifiée », c'est-à-dire que l'objectif prescrit n'est alors plus le bon état, mais le bon potentiel ${ }^{3}$, qui consiste en un objectif adapté ;

- des reports d'échéance du bon état à 2021 ou 2027, tout en garantissant que l'état de la masse d'eau ne se détériore pas davantage ;

- des objectifs environnementaux moins stricts que le bon état.

\footnotetext{
2 Ce n'est que lorsque les objectifs de 2015 apparaîtront «techniquement réalistes » que la nécessité d'un report des délais, en raison de coûts disproportionnés, sera examinée.

3 Il s'agit alors du meilleur objectif écologique atteignable compte tenu des contraintes morphologiques.
} 
Il s'agit donc bien, théoriquement, d'utiliser l'analyse économique pour l'aide à la décision publique en évaluant les risques de coûts disproportionnés que pourrait générer l'application de cette politique environnementale pour la société. Toute la question est de savoir comment procéder pour juger du caractère disproportionné du coût, puis comment utiliser l'indicateur de coût disproportionné ainsi conçu dans la décision publique.

Malgré l'enjeu de ces évaluations, les méthodes à mobiliser pour prouver le caractère disproportionné des coûts ne sont pas précisées dans les textes. Ces dérogations permettent pourtant de faire bouger le curseur du niveau d'ambition pour un bassin donné et ainsi, pour un État, de jouer sur ce critère en vue d'introduire des biais de concurrence entre les États membres. Par ailleurs, à l'échelle du bassin, voire plus localement, les actions censées permettre d'atteindre le bon état des eaux impliquent bien souvent de modifier des infrastructures (renforcement de la capacité de traitement d'une station d'épuration des eaux usées...), des aménagements (suppression d'un barrage sur une rivière pour rétablir la libre circulation des poissons...), des pratiques (diminution forte, voire suppression de certains intrants en agriculture, dans certaines zones jugées prioritaires pour l'eau...).

Ces aménagements sont suffisamment importants pour être contestés par les acteurs locaux. Il paraît donc assez évident que l'étude du caractère disproportionné du coût du projet est susceptible de rendre compte des tensions existantes entre l'ambition des objectifs environnementaux et l'importance des changements à entreprendre pour les atteindre.

La question de la méthode la plus appropriée pour juger du caractère disproportionné du coût a fait l'objet de débats entre les directeurs de l'eau des États membres : analyse coûts-bénéfices ou évaluation de la capacité financière des acteurs à supporter le coût du projet. Dans ce débat, les pays les moins riches ont globalement défendu le recours à "l'analyse de la capacité financière ", tandis que les autres défendaient l'utilisation d'une analyse coûts-bénéfices. La France, via le ministère de l'Environnement, a adopté à l'époque une posture "pro-analyse coûts-bénéfices» qui ne reflétait pas l'avis des économistes des agences, lesquels avançaient que :

1. pour justifier une dérogation de délai, donc un étalement du projet, sans pour autant remettre en cause l'objectif, une analyse coûts-bénéfices semblait a priori moins appropriée qu'une analyse de la capacité financière circonstanciée des acteurs et des financeurs ;

2. réaliser des analyses coûts-bénéfices sur toutes les masses d'eau pourrait avoir pour effet de rabaisser de beaucoup l'ambition de la directive à l'horizon 2015 ; en effet, les économistes des agences subodoraient déjà l'impossibilité de chiffrer tous les bénéfices, et donc leur prévisible faiblesse comparée aux coûts ;

3. réaliser des centaines d'analyses coûts-bénéfices de manière nécessairement "industrielle ${ }^{4}$ » et peu contextualisée aurait peu de sens.

La poire a finalement été coupée en deux et, en France, en référence au code de l'environnement ${ }^{5}$, un guide méthodologique a été publié en octobre 2009 pour détailler la méthode à utiliser :

- il s'agit au préalable d'identifier les masses d'eau (ou groupes de masses d'eau) susceptibles de présenter des coûts disproportionnés, en comparant le coût d'atteinte des objectifs de bon état à l'horizon 2015 aux capacités financières du territoire (avec des critères de jugement définis au niveau du bassin) ;

- sur ces masses d'eau "à risque de disproportion", après s'être assuré que les dérogations ne peuvent être obtenues pour cause de faisabilité technique ou de conditions naturelles, il s'agit de réaliser une analyse coûts-bénéfices ;

- dans le cas où les bénéfices sont inférieurs à $80 \%$ des coûts, la justification est jugée suffisante (le coût est considéré comme disproportionné) ;

- si les bénéfices sont supérieurs à $80 \%$ des coûts, il est possible d'approfondir l'analyse en répartissant les coûts par secteur, et de mettre cela en regard des capacités financières des acteurs, en tenant compte des subventions accordées à ces derniers, pour justifier d'une éventuelle disproportion.

\section{Le système des agences de l'eau}

Pour pouvoir appréhender la question des coûts disproportionnés, il importe de clarifier le cadre de gouvernance dans lequel l'usage de cette analyse a été réalisé. Les agences de l'eau sont des établissements publics gérant des taxes affectées liées à l'eau. Ces organismes ont été créés par la loi sur l'eau du 16 décembre 1964 dans un contexte d'économie planifiée et centralisée. Nommées "agences financières de bassin » jusqu'en 1992, leur mission originelle a été de coordonner la politique de l'eau à une échelle cohérente du point de vue des écoulements de surface - le bassin versant -, afin de restaurer une qualité de l'eau mise à mal par la croissance urbaine et industrielle. Leur travail consiste à prélever des taxes proportionnelles à la pollution et aux prélèvements sur la ressource (selon le principe " pollueur-payeur ») et à en utiliser le produit afin de verser

\footnotetext{
4 C'est-à-dire de manière standardisée, en grand nombre, par contraste avec une analyse qui serait produite sur mesure.

5 Décret n²005-475 accompagné de la circulaire DCE 2006/ 17 relative à l'élaboration, au contenu et à la portée des programmes de mesures.
} 
des aides aux porteurs de projets améliorant la gestion de l'eau (collectivités voulant construire une station d'épuration des eaux usées, par exemple) en vue d'aboutir à une meilleure gestion de l'eau à l'échelle des bassins versants. Les projets aidés sont retenus sur la base de critères définis dans un programme pluriannuel. L'originalité de ce système réside notamment dans le fait que les programmes pluriannuels des agences, le choix des projets à retenir, le niveau des aides apportées, comme celui des taxes perçues, sont définis et validés par un "comité de bassin", forme de "parlement de l'eau » qui rassemble des représentants des collectivités, des usagers et de l'État, et qui se réunit non seulement en assemblée plénière, mais donne également lieu à des instances et groupes de travail thématiques ou locaux. $C^{\prime}$ est dans ce cadre que sont définis les objectifs du SDAGE, en particulier les objectifs de qualité assignés aux masses d'eau du bassin, pour être ensuite soumis à validation du préfet coordinateur de bassin. C'est aussi dans ce cadre que les évaluations économiques permettent de justifier de coûts disproportionnés.

Nous allons à présent rendre compte de l'expérience des économistes des agences de l'eau en matière d'usage de l'analyse coûts-bénéfices, quasiment non utilisée dans la pratique des agences jusque-là, pour définir les objectifs de qualité des eaux, puis nous discuterons cette démarche.

\section{Les méthodes d'évaluation utilisées}

Il existe plusieurs méthodes pour évaluer les bénéfices monétaires associés à la restauration du bon état des eaux terrestres.

Pour chiffrer les bénéfices, on a coutume de distinguer différentes valeurs (Chevassus-au-Louis et al., 2009 ; EPA, 2009) : les valeurs d'usage (direct, comme la pêche, ou indirect, via des services comme la régulation des inondations, l'augmentation de la biodiversité) et de non-usage (legs aux générations futures, valeur altruiste, valeur d'existence de l'environnement). Parmi les valeurs d'usage, on distingue les usages " marchands " (par exemple, la production d'eau potable), relativement faciles à chiffrer car donnant lieu à des échanges, et des usages " non marchands » (par exemple, la baignade), plus difficiles à chiffrer.

Pour approcher les valeurs non marchandes, on distingue trois types de méthodes d'évaluation (Barbier et al., 2009) : les méthodes basées sur les coûts (coût des dommages évités, coûts de remplacement, coûts substituts, coût de restauration, impact sur la productivité) ; les méthodes des préférences révélées (prix hédoniques [ou hédonistes], coûts de transport, prix de marché) ; les méthodes de préférences déclarées (consentements à payer établis sur la base d'évaluations contingentes et d'analyses conjointes). Ces trois méthodes peuvent également être mixées pour obtenir des raffinements dans les évaluations (Appéré, 2004). Les méthodes de préférences déclarées sur des marchés fictifs sont les seules permettant actuellement d'appréhender les valeurs de non-usage (Levrel et al., 2012). Elles seules permettent en effet de demander à des individus combien ils consentiraient à payer ou à recevoir pour, respectivement, la conservation ou la destruction d'une composante de la nature, qui ne génère aucun bénéfice direct ou indirect mais a une valeur par le fait que les individus expriment un attachement à l'existence même de cette composante. Il s'agit aussi des méthodes les plus pratiques à mettre en œuvre car elles permettent, à partir d'une simple enquête, de récupérer des informations sur tous les types de valeurs issus de la protection d'un écosystème. Ces méthodes sont cependant aussi celles qui sont les plus critiquées du fait de leur caractère très peu robuste et des niveaux d'incertitudes qui entourent les estimations auxquelles elles donnent lieu (Milanesi, 2011).

Une fois les valeurs de l'ensemble des bénéfices estimées, en distinguant différents services rendus et en évitant les redondances pour pouvoir les sommer, elles sont agrégées en vue d'obtenir ce que l'on nomme une "valeur économique totale » (Chevassus-au-Louis et al., 2009 ; CGDD, 2011).

Nous avons utilisé plusieurs méthodes pour évaluer les bénéfices générés par la restauration du bon état des eaux : la méthode des coûts évités a été appliquée à travers l'évaluation monétaire de la diminution des coûts de traitements et les évaluations contingentes (et plus marginalement des méthodes des coûts de transport) ont permis d'évaluer l'accroissement de la satisfaction des usagers.

La réalisation d'un grand nombre d'analyses coûtsbénéfices a conduit à avoir recours à des transferts de bénéfices unitaires (Brouwer, 2000). La méthode des transferts de bénéfices consiste à utiliser sur un site donné une valeur monétaire élaborée sur un autre site, si possible similaire sur les plans biophysique et socioéconomique, quitte à ajuster les valeurs en conséquence. Elle est très utilisée aujourd'hui car elle permet de réaliser des économies substantielles dans les processus d'évaluation des services écosystémiques.

Dans le cas de la DCE, pour faciliter la réalisation des analyses coûts-bénéfices et en diminuer le coût de mise en œuvre, le ministère de l'Environnement a mis à la disposition des agences de l'eau un tableur permettant de transférer facilement les valeurs unitaires obtenues dans des études françaises reconnues qui relient le changement de qualité environnementale à un changement de valeur. L'idée était notamment de ne pas alourdir la procédure avec la réalisation d'enquêtes de terrain destinées à évaluer les consentements à payer, pour monétariser 
les bénéfices non marchands des projets de reconquête du bon état des eaux (MEDD, 2005).

Pour utiliser ce tableur, les économistes des agences en charge de l'évaluation monétaire commencent par indiquer le type de milieu concerné (cours d'eau, plan d'eau, eau côtière ou souterraine), puis sélectionnent le type de bénéfices attendus (par exemple, les poissons sauvages, initialement peu présents, qui vont pouvoir vivre et se reproduire dans le milieu grâce au changement d'état). En fonction des critères saisis, l'outil sélectionne les bénéfices les plus proches (le premier critère de similarité étant la taille du cours d'eau), avec souvent une valeur minimale et maximale, que l'économiste peut valider ou refuser. Ces valeurs unitaires sont appliquées à la population censée bénéficier des services écosystémiques offerts par les cours d'eau évalués. En multipliant le taux unitaire par la population concernée, on obtient les bénéfices associés à certains services écosystémiques. Ainsi les consentements à payer des kayakistes issus de l'étude d'origine sont appliqués au nombre d'usagers kayakistes estimés sur le tronçon de cours d'eau considéré. La méthode est la même pour les pêcheurs, les baigneurs, les promeneurs. Lorsque ces données sont incertaines, soit parce qu'elles sont disponibles à des échelles moins fines, soit que leur estimation à l'échelle considérée comporte elle-même une incertitude, des fourchettes d'estimation sont utilisées. Parallèlement, l'économiste saisit les données relatives aux coûts du projet (y compris la durée de vie des investissements, les coûts de fonctionnements), éventuellement à l'aide de «fourchettes » de coûts. Pour finir, une comparaison des coûts et des bénéfices actualisés est effectuée, comme l'illustre l'exemple dans l'encadré (Large, 2008).

Nous proposons maintenant de présenter les résultats à l'échelle nationale, sachant que les analyses ont été conduites au niveau de chacun des sept grands bassins ${ }^{6}$ qui constituent le territoire métropolitain.

Sur l'ensemble de la France, qui compte 11523 masses d'eau « de surface ${ }^{7}$ ", la démarche de sélection préalable sur la base des critères de capacité financière des acteurs, tel que préconisé par le guide national, a conduit au final à réaliser 710 analyses coûts-bénéfices à l'échelle de masses d'eau ou de groupes de masses d'eau, donc de petits bassins versants. Les analyses dont il est question ici ont majoritairement été conduites de manière « industrielle » pour en limiter le coût, en utilisant les données disponibles sur les bassins versants considérés et, pour les valeurs non marchandes, en transférant comme expliqué auparavant des consentements à payer unitaires issus d'études réalisées sur des masses d'eau de caracté-

\footnotetext{
6 Ces sept bassins sont : Seine-Normandie, Loire-Bretagne, Rhin-Meuse, Artois-Picardie, Adour-Garonne, Rhône-Méditerranée et Corse ( 2 bassins, mais une seule agence).

7 À distinguer des masses d'eau souterraine.
}

ristiques relativement proches, grâce au tableur du ministère de l'Environnement décrit plus haut.

Le tableau ci-après récapitule les démarches adoptées au niveau des sept grands bassins hydrographiques (le bassin de la Corse étant groupé avec celui de RhôneMéditerranée).

Ce tableau montre que si la méthode définie au niveau national a globalement été suivie, chacun des six bassins l'a déclinée différemment. Certains ont utilisé l'analyse coûts-bénéfices comme un moyen de justification a posteriori et l'ont conduite sur un grand nombre de masses d'eau de manière « industrielle », d'autres ont mené un nombre très réduit d'analyses approfondies plus ou moins mobilisées pour la décision; d'autres encore ont opté pour une démarche mixte et enfin, quelques bassins ont jugé préférable de mettre le moins possible en avant le critère économique comme motif de dérogation.

\section{Discussion}

En théorie, la mobilisation d'une analyse coûts-bénéfices ex ante s'explique par une volonté de rationalisation des choix en politique publique. Il s'agit, notamment dans le cas de politiques environnementales qui peuvent s'avérer onéreuses, et dont l'intérêt n'est pas toujours évident, de comparer les coûts payés par la société aux bénéfices qu'elle tire du projet. Cette démarche semble relever du bon sens et constituer un garde-fou nécessaire pour éviter des projets dont les coûts sociaux seraient supérieurs aux bénéfices sociaux.

Cependant, elle soulève rapidement des problèmes difficilement surmontables, comme en témoigne la position de la Commission européenne sur la question. En effet, cette dernière était en principe tenue de réaliser une étude coûts-bénéfices de la DCE (que les États membres ont d'ailleurs réclamée). Elle n'a pas été menée à ce niveau, sous prétexte que les plans de gestion attendus tous les six ans au niveau des bassins hydrographiques sont censés comporter des analyses économiques (De Abreu, 2001), l'autre argument avancé par la Commission étant que « toute estimation chiffrée complète des coûts et des bénéfices en termes financiers serait au mieux peu fiable et au pire trompeuse ou carrément fausse " (Commission européenne, 1997 $)$. Dans quelle mesure ce jugement de la Commission, porté sur une analyse à l'échelle européenne, s'applique-t-il également à des analyses effectuées à des échelles plus fines ?

\footnotetext{
8 Depuis, une tentative d'analyse coûts-bénéfices a cependant été réalisée sur la base des analyses conduites par les bassins, qui conclut notamment à la difficulté de l'exercice étant donné les différences de méthodes employées localement (Matthei $\beta$ et al., 2012).
} 


\section{Encadré : Exemple d'analyse coûts-bénéfices réalisée pour le bassin de « Béthunes et Arques » (2008)}

Le bassin « Béthune et Arques » regroupe trois masses d'eau et s'étend sur $357 \mathrm{~km}^{2}$. Il comprend un fleuve côtier situé en Haute-Normandie, débouche sur deux plages et intègre un site de loisir en amont. Son état initial est médiocre, notamment en raison de la faible diversité des poissons, et du fait d'un excès de phosphore provenant de l'agriculture, déclassant la qualité de l'eau. Sur ce cas, les mesures visant à reconquérir le bon état des eaux en 2015 sont chiffrées à 235 millions d'euros, 85 \% correspondant à des coûts récurrents (coûts d'entretien de la rivière) et $15 \%$ à des investissements (amélioration du traitement des eaux usées d'une station d'épuration).

Les bénéfices unitaires non marchands issus des études de cas dont les caractéristiques semblaient les plus proches (sur la base des critères retenus par l'outil du ministère de l'Environnement) du bassin considéré sont présentés dans la figure ci-dessous (avec la rivière de référence : Gardon ou Loir).

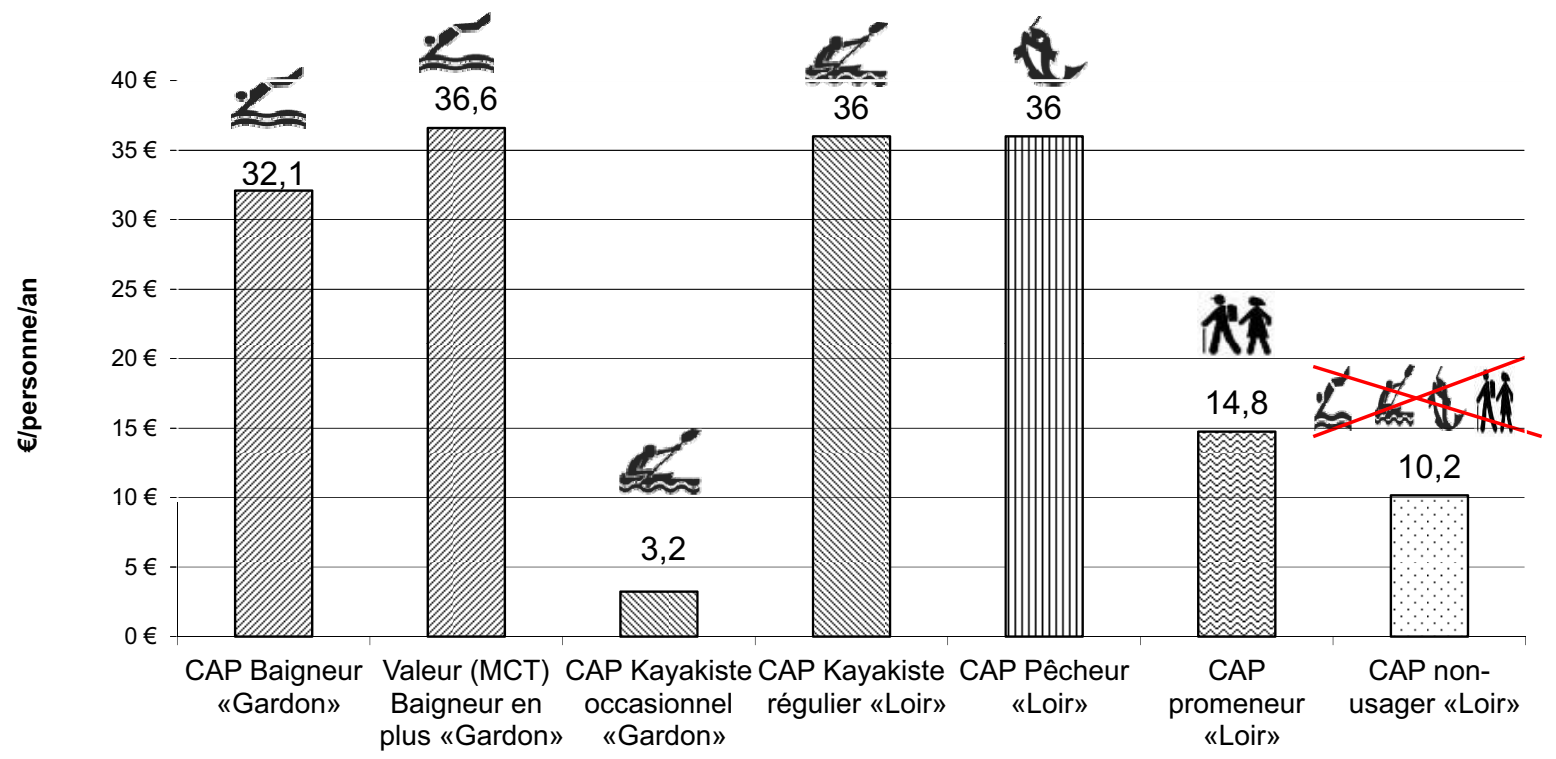

Fig. 2. Consentements à payer (CAP) et valeur issue de la méthode des coûts de transport (MCT) de différents types d'usagers (et non-usagers) retenus dans le cas présenté (Large, 2008).

Pour choisir les assiettes auxquelles appliquer les consentements à payer unitaires issus de transferts de valeurs, et ainsi extrapoler les bénéfices associés aux services écosystémiques restaurés à travers les actions visant à atteindre le bon état des eaux, il est nécessaire de pouvoir utiliser une population de référence qui bénéficie de ces services écosystémiques. Dans notre exemple, c'est la population du bassin qui a été retenue par l'économiste, en utilisant les estimations sur le nombre d'usagers (kayakistes, baigneurs...) aux échelles régionales, départementales ou à l'échelle de sites, faute de données précises sur l'intérêt suscité par ce cours d'eau en lien avec un périmètre géographique.

Ce choix n'a suscité aucune réaction. Il semble réaliste, même sur la base d'études approfondies, comme le montre celle pilotée par le Commissariat général au développement durable sur les zones humides (CGDD, 2011) de circonscrire exactement la zone d'intérêt suscitée par un milieu ou un cours d'eau.

En définitive, la somme des bénéfices actualisés sur la période 2010-2040 (fondée sur la durée de vie des équipements) semble inférieure aux coûts : ils sont estimés à 18,2 millions d'euros, quand le coût se monte à 235 millions d'euros.

Comme indiqué dans le tableau suivant, la méthode et le résultat présentés ici sont représentatifs de la majorité des analyses coûts-bénéfices réalisées dans le cadre de la DCE. Cependant, on peut d'ores et déjà noter qu'une petite proportion $(0,4 \%)$ des analyses coûts-bénéfices n'a pas eu recours au transfert de bénéfices, contrairement au cas exposé dans cet encadré, mais a plutôt reposé sur des enquêtes de consentement à payer réalisées sur site (voir dans le tableau, le cas « Loire-Bretagne »). Ce choix coûteux et chronophage est effectué lorsque le site présente un intérêt particulier et que le projet dont il fait l'objet doit donner lieu à un débat dépassant le cercle technique. 
Pour répondre à cette question, nous souhaitons discuter plusieurs points qui démontrent le caractère discutable du statut de ces évaluations.

\section{L'impact des évaluations sur les processus de dérogation}

La DCE impose qu'une demande de dérogation soit faite au regard de raisons "techniques ", "naturelles " ou « économiques ». Pour des raisons d'interprétation de texte, de compromis et de pragmatisme, l'analyse coûtsbénéfices a été considérée en France comme l'argument central mis en avant dans ces demandes de dérogations, même si en termes de rationalité économique l'analyse des capacités financières semblait plus appropriée à des dérogations de délai qui correspondent à un besoin d'étalement dans le temps du financement du projet politique. Des impératifs de proportionnalité du travail à fournir et de coûts ont induit la réalisation d'analyses coûts-bénéfices « industrielles » basées sur des transferts de bénéfices par rapport à des analyses coûts-bénéfices « de terrain » qui auraient pu ouvrir la porte à des considérations plus subtiles, éventuellement multicritères, et à de réelles discussions politiques à la lumière des éléments dégagés. Les analyses coûts-bénéfices «industrielles» ont finalement été considérées comme un moyen pratique de fournir à peu de frais les justifications demandées, quitte à ce que le choix de l'objectif des masses d'eau repose en réalité sur d'autres considérations (plus politiques, d'acceptabilité, de compromis...). $C^{\prime}$ est pourquoi il est permis d'affirmer que les analyses coûts-bénéfices ont été le plus souvent un outil de justification ex post de choix politiques déjà validés et non pas un outil de rationalisation ex ante de la décision publique.

$\mathrm{Au}$ regard des estimations réalisées par les agences de l'eau, il apparaît très facile d'utiliser ces analyses coûts-bénéfices pour justifier une dérogation de délai ou d'objectif, dans la mesure où, le plus souvent, les bénéfices ne pèsent pas lourd dans la balance : les trois quarts de ces 710 analyses coûts-bénéfices présentent des bénéfices largement inférieurs aux coûts (Tab.). Par conséquent, si cette méthode avait été utilisée pour fixer le niveau d'ambition de l'ensemble des bassins, celui-ci aurait été beaucoup plus faible que l'objectif considéré comme «acceptable» pour la collectivité, dans la mesure où il a été voté par les comités de bassin.

Ainsi, sur le bassin Artois-Picardie où des analyses relativement fines sur les coûts-bénéfices ont été conduites, les services de l'agence, en accord avec ceux de l'État, ont choisi de ne faire reposer aucune dérogation sur des critères uniquement économiques, du fait de leur caractère «discutable ». Si aucune dérogation ne reposait uniquement sur un critère économique, c'est parce qu'il n'apparaissait pas pertinent que ce seul critère soit décisif. Il a été privilégié une approche où le critère économique était mixé à un critère technique et/ou environnemental. En l'état, les analyses économiques auraient été suffisantes pour justifier d'une dérogation, mais comme évoqué, c'est le parti pris de la mixité qui a été retenu.

\section{Diversité des méthodes et des manières de conduire les évaluations : des sources d'incertitudes}

Un premier point qui pose problème est la méthode retenue pour réaliser les évaluations. En effet, s'il existe deux méthodes qui sont considérées comme relativement peu robustes par les économistes, ce sont les évaluations contingentes et les méthodes de transfert de bénéfices (TEEB, 2010 ; Levrel et al., 2012 ; Milanesi, 2011). La recherche en économie souligne ainsi l'insuffisance de ces méthodes à l'heure actuelle (Salles, 2010) et leur nécessaire perfectionnement grâce à de nouvelles recherches. Or, ces deux méthodes sont celles qui ont été utilisées dans les estimations réalisées par les agences de l'eau.

Ensuite, si les six agences de l'eau françaises ont globalement suivi le guide national, les stratégies de mise en œuvre ont différé sur plusieurs aspects, comme le montre le tableau : sur le fait même de réaliser des évaluations monétaires, sur l'emploi (ou non) du tableur, sur le choix de la population à retenir pour l'extrapolation des valeurs, et sur la manière d'inscrire ces évaluations dans un processus décisionnel.

Enfin, certains services écosystémiques fournis par les masses d'eau sont difficilement chiffrables car éminemment subjectifs, peu commensurables et/ou sujets à de nombreuses incertitudes. Tel est le cas des valeurs d'inspiration, d'existence, de la valeur scientifique, du bien-être, mais également de la résilience des écosystèmes, qui dépend de la biodiversité et s'évalue sur un temps très long et des maillages complexes. Il y a donc des avantages induits par un bon état des eaux qui ne peuvent être traduits par des valeurs économiques. $\mathrm{Au}$ total, on a un déficit certain de connaissances, qui nous conduit à ne pouvoir appréhender que partiellement les bénéfices environnementaux.

Par ailleurs, la variabilité des valeurs unitaires de consentement à payer selon les études, et le fait d'avoir décliné différemment, selon les bassins, la manière de conduire ces analyses coûts-bénéfices (calculs de masse à partir de l'outil du ministère, études détaillées...) montrent en soi que ces conventions ne sont pas répliquables comme pourraient l'être des calculs biophysiques ou comptables. Cela nous amène à souligner maintenant 


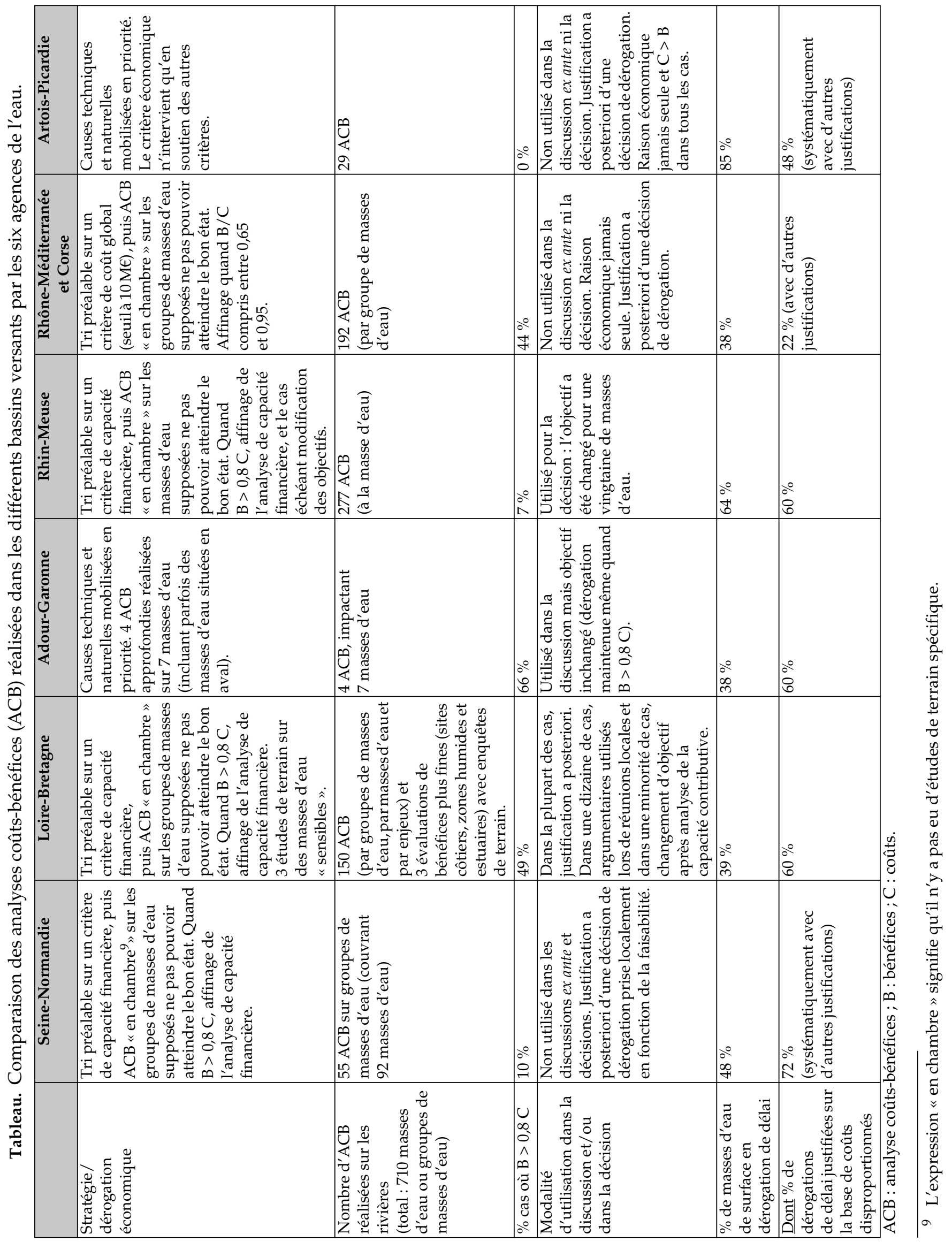


l'élément le plus discutable sur le plan technique : la latitude laissée aux économistes pour choisir arbitrairement l'assiette de population à laquelle sera affecté le consentement à payer.

\section{Le rôle-clé de la population de référence retenue}

Aucune loi ne permet de délimiter de manière rigoureuse les " périmètres d'intérêt » de la zone considérée et donc la population de référence à prendre en compte pour réaliser les évaluations des bénéfices associés à la restauration du bon état des masses d'eau.

Prenons l'exemple de deux bassins versants en Languedoc-Roussillon, le Vidourle, qui compte 42000 habitants, et le Lez-Mosson, qui compte 414000 habitants (la ville de Montpellier est incluse dans ce bassin). Pour ces deux bassins, le projet de reconquête du bon état présente un coût équivalent et, à l'échelle du bassin entier, un intérêt similaire en termes de gain écologique. Dans le premier cas, il s'agit principalement de restaurer l'espace de liberté des cours d'eau et du littoral et de lutter activement contre les pollutions agricoles ; dans le deuxième, l'objectif est de lutter contre les pollutions urbaines (notamment liées aux écoulements pluviaux et aux sites pollués). Les analyses coûts-bénéfices concluent que pour le Vidourle, le projet de reconquête du bon état des eaux est chiffré à $27,6 \mathrm{M} €$, tandis que les bénéfices ne pèsent que 13,4 M€ malgré le tourisme important et une activité développée de canoë-kayak. Le coût du projet est donc jugé disproportionné. Pour le Lez-Mosson, le projet est chiffré à 27,4 M€ et les bénéfices à $125 \mathrm{M} €$ du simple fait de la population de Montpellier. Les coûts ne sont donc pas jugés disproportionnés. En définitive, si les masses d'eau des deux bassins versants ont fait l'objet de dérogations, l'argument des coûts disproportionnés a été avancé seulement dans le cas du Vidourle, d'autres arguments (techniques, naturels) étant avancés dans le cas du Lez-Mosson.

Cet exemple nous montre des écarts de valeurs importants du fait de différences de populations de référence, il semble donc nécessaire de disposer d'une méthode précise pour déterminer la population concernée par la restauration du bon état des eaux sur différents bassins versants.

Des études approfondies menées par le Commissariat général au développement durable sur la valorisation des services rendus par les zones humides (CGDD, 2013, p. 3-4) indiquent bien que « la valeur finale du service dépendra du nombre d'individus à qui l'on attribue ce consentement à payer » et $\mathrm{qu}^{\prime}$ «il est souvent complexe empiriquement de déterminer l'étendue exacte de cette zone d'influence». Les auteurs concluent : «Plus que les consentements à payer unitaires, c'est sur les méthodes d'identification des populations concernées par chacun des services rendus par les zones humides des sites étudiés [...] que doivent porter les efforts des prochains travaux de monétarisation ». Mais rien n'est moins simple. Ainsi, une enquête de consentement à payer (CAP) pour restaurer le marais du Cotentin et du Bessin, dans des lieux plus ou moins éloignés de ce marais (CGDD, 2011), souligne que les écarts entre les CAP ne résultent pas seulement de la distance au site (ce qui validerait ou faciliterait l'identification d'une population de référence) mais également d'un grand nombre de paramètres tels que la taille de la ville d'appartenance, les critères socioprofessionnels ou encore la pratique d'activités en rapport avec l'environnement... C'est dire la difficulté qui existe à vouloir établir les populations de référence.

\section{Les utilisations stratégiques inattendues des évaluations monétaires}

Il convient, au-delà des limites mentionnées précédemment, de considérer les effets pervers potentiels de ces évaluations lorsqu'elles commencent à être utilisées dans le cadre de la mise en œuvre de politiques publiques environnementales. Nous en avons relevé au moins deux. Tout d'abord, le retournement de l'outil de preuve : après l'adoption du SDAGE (qui comprend les objectifs sur les masses d'eau à l'horizon 2015), des industriels situés sur des masses d'eau jugées globalement aptes sur les plans technique, naturel et financier à atteindre le bon état en 2015 et qui n'avaient pas fait l'objet d'analyses économiques, ont demandé à ce que des analyses coûts-bénéfices soient réalisées afin de prouver que les bénéfices du projet de restauration du bon état sur les masses d'eau les concernant n'étaient pas à la hauteur des coûts. Il est aisé d'imaginer, étant donné le contexte d'incertitude, la fragilité de la méthode et le faible crédit accordé à ces analyses, la difficulté dans laquelle ont été plongées les agences de l'eau concernées, sachant que l'analyse coûts-bénéfices avait été utilisée pour justifier des dérogations, et non pour accompagner des objectifs de qualité. Cela pose d'autant plus de problèmes que les agences de l'eau fonctionnent sur la base d'un schéma de gouvernance qui donne presque autant de pouvoir aux usagers de l'eau (y compris les industriels) qu'à l'État. Cette méthode de justification pourrait donc être utilisée " à charge " par des acteurs ne souhaitant pas que les projets de restauration soient adoptés. D'un outil utilisé comme une pseudo- justification a posteriori, on passe à un outil de lutte contre la gestion durable de l'eau par des acteurs économiques ne souhaitant pas faire plus d'efforts. Une telle situation pourrait déboucher sur un embourbement judiciaire si un acteur privé décidait de faire un recours en justice, comme ce fut le cas aux États-Unis pour les méthodes de calcul des compensations en cas de marée noire, lorsque les méthodes 
d'évaluation monétaires étaient utilisées au cours des années 1990 (Thompson, 2002).

Autre effet pervers, plus global : un référentiel normatif économique, dont le caractère discutable montré précédemment vient contrebalancer les objectifs fixés par une loi, risque, si le raisonnement est poussé à l'extrême, de vider cette loi de son contenu. Une utilisation de l'analyse coûts-bénéfices pour fonder directement un choix politique (et non pour justifier a posteriori un choix effectué sur la base d'autres éléments) pose finalement la question du risque qu'il y a à produire des chiffrages par rapport à l'enjeu environnemental du fait de la sous-estimation déjà évoquée ou du manque de crédibilité de certaines méthodes.

\section{Des méthodes de calcul alternatives}

Ces difficultés pourraient conduire à préconiser l'évaluation de la biodiversité par la méthode des coûts de maintien ou d'atteinte du bon état des eaux, plus tangible et circonstanciée que par celle des bénéfices (Levrel et al., 2012). En effet, le calcul du coût des actions à réaliser est beaucoup moins discutable puisque fondé sur de simples dépenses affectées à des actions précises. Cependant, cela ne peut se concevoir dans le cadre des analyses coûts-bénéfices que la DCE demande de produire pour justifier des dérogations, puisque l'exercice consiste précisément à comparer les coûts aux avantages. Une autre piste est de s'intéresser aux bénéfices à partir d'indicateurs monétaires, mais aussi d'indicateurs d'accès, écologiques, sanitaires et de tout autre indicateur pouvant avoir du sens pour la population du territoire concerné. Il n'est en effet pas obligatoire d'avoir recours à un seul étalon pour faire des comparaisons entre les coûts et les bénéfices. En Angleterre, où tout nouveau projet de loi doit être étayé par une analyse coûts-bénéfices, il est admis que l'on peut s'appuyer sur tous types d'indicateurs pour justifier des bénéfices de l'action publique, ces derniers ayant avant tout pour intérêt de venir alimenter un débat public sur la question des coûts et des bénéfices pour la société, et non de venir justifier un choix rationnel d'un strict point de vue économique (Pearce et al., 2006).

\section{Conclusion}

Cet article offre une illustration des tensions qui peuvent exister entre des référentiels légaux et économiques concernant la mise en œuvre d'une politique publique environnementale. Si ces deux référentiels peuvent fournir les bases d'un débat public sur la pertinence des actions de restauration du bon état des eaux, il est important de questionner leur qualité.

Il semble, au regard du retour d'expérience des économistes des agences de l'eau, que la robustesse du référentiel économique peut être questionnée. En effet, si les coûts de restauration du bon état des eaux sont évaluables à partir de simples mesures comptables des dépenses, il n'en va pas de même pour l'évaluation des bénéfices qui vont être mis en balance avec ces coûts pour évaluer le caractère plus ou moins disproportionné de ces derniers. L'évaluation des bénéfices générés par la mise en œuvre d'actions de restauration du bon état dépend en effet de deux paramètres qui font fortement varier les estimations :

- la méthode de l'évaluation contingente, la plus discutée par la communauté des économistes mais aussi la moins coûteuse à mettre en œuvre si on utilise des transferts de valeurs ;

- le périmètre de la population à laquelle vont être appliqués les coûts unitaires et, conjointement, le faible niveau de connaissance du nombre d'usagers exact des services écosystémiques générés par la restauration du bon état des eaux.

Ces deux paramètres créent des espaces d'incertitudes qui laissent place à une diversité d'interprétations possibles et peuvent finalement être utilisés de manière stratégique par des acteurs qui auraient intérêt à faire peser l'évaluation dans un sens ou dans un autre. Ce type de situation a déjà été à l'origine de nombreuses controverses aux États-Unis dans le cadre notamment de l'évaluation des dommages associés aux marées noires, l'exemple le plus emblématique étant l'Exxon Valdez: d'une évaluation à une autre, les analystes obtenaient des estimations variant de un à dix. De cela ont découlé de longues procédures, tout aussi coûteuses pour l'État que pour les entreprises polluantes, et finalement l'abandon de ces méthodes pour monétariser les dommages environnementaux, les cours de justice leur préférant une évaluation des coûts de restauration des écosystèmes comme approximation de la valeur des dommages (Thompson, 2002). Évidemment, dans notre cas d'étude, ce type d'évaluation n'a pas de sens puisqu'il s'agit ici d'évaluer le coût disproportionné de la mise en œuvre d'une politique environnementale de l'eau. Pour autant, elle interroge sur la capacité des analyses coût-bénéfices à fournir un cadre méthodologique adéquat pour justifier de ces coûts disproportionnés.

\section{Perspectives}

En définitive, pour avoir une vision globale de l'intérêt d'un projet pour la collectivité, il nous semble préférable de recourir à un affichage qualitatif des bénéfices non marchands et de s'efforcer de mener un réel débat 
considérant l'ensemble des valeurs et pas seulement celles qui sont traduites en euros (par exemple, l'intérêt pour la population locale de la remise en baignade d'une portion de rivière). Les premiers retours d'expérience de l'utilisation par d'autres États membres de l'évaluation monétaire de la nature pour la DCE, qui nous reviennent par exemple à travers le projet de recherche européen ESAWADI ${ }^{10}$, montrent, sur les cas portugais et allemand considérés, une réticence similaire à l'usage de l'analyse coûts-bénéfices, à laquelle sont préférées des méthodes telles que l'analyse multicritère ou «l'approche Leipzig » qui consiste à ne pas monétariser les bénéfices et à faire du " semi-qualitatif » basé sur le dire d'expert (Blancher et al., 2013).

Concrètement, il semble important :

- de qualifier les bénéfices non marchands (grandeurs biophysiques, nombre d'usagers...) ;

- de faire apparaître les valeurs qui leur sont attachées, au sens premier du terme plutôt que monétaires (valeur d'usage, valeur patrimoniale, attachement, références historiques, sentimentales...) ;

- d'utiliser l'évaluation économique pour bâtir des plaidoyers au service des lois environnementales, à partir de coûts et de bénéfices observables à l'image du rapport du Commissariat général au dévloppement durable sur le coût des pollutions diffuses, dont les principaux messages retenus concernent les économies de coûts de traitement de l'eau ; c'est aussi le cas des comparaisons de bénéfices nets pour la collectivité de plusieurs scénarios de développement en termes de résultats économiques, d'économies budgétaires et d'emplois (Garambois et Devienne, 2010) ;

- de considérer, comme le soulignent également d'autres auteurs (Laurans et al., 2001), les évaluations économiques comme des "processus sociaux de constructions de valeurs ", à l'inverse de méthodes standardisées supposant que l'évaluation peut révéler des valeurs préexistantes.

Pour cela, il semble que les politiques doivent prendre le relais de manière à garantir que les arbitrages soient le fruit d'un processus démocratique et de négociations, et pas uniquement d'une analyse coûtsbénéfices. C'est aussi ce que conclut Salles (2010) quand il écrit que l'enjeu de la conservation de la biodiversité (également inclus, soulignons-le, dans la gestion de l'eau et la DCE) est trop grave pour être confié aux seuls économistes.

${ }^{10}$ Le projet ESAWADI (Utilising the ecosystem services approach for water framework directive implementation), réalisé dans le cadre de l'appel à projet de recherche sur la gestion intégrée des ressources en eau IWRM-NET, a analysé la valeur ajoutée de l'approche d'évaluation des services écosystémiques pour la décision dans le cadre de la DCE, en particulier sous un angle économique.

\section{Références}

Appéré, G., 2004. L'évaluation des actifs à usage récréatif. La méthode contingente des coûts de transport, Revue d'Économie Régionale \& Urbaine, 1, 81-106.

Barbier, E.B., Baumgärtner, S., Chopra, K., Costello, C., Duraiappah, A., Hassan, R., Kinzig, A., Lehmann, M., Pascual, U., Polasky, S., Perrings, C., 2009. The valuation of ecosystem services, in Naeem, S., Bunker, D.E., Hector, A., Loreau, M., Perrings, C., (Eds), Biodiversity, ecosystem functioning $\mathcal{E}$ human wellbeing, Oxford, Oxford University Press, 248-262.

ESAWADI, 2013. Utilizing the ecosystem services approach for water framework directive implementation. Synthesis Report, http://www.esawadi.eu/IMG/pdf/ Esawadi_final_synthesis_july2013.pdf.

Brouwer, R., 2000. Environmental value transfer. State of the art and future prospects, Ecological Economics, 32, 1, 137-152.

Chevassus-au-Louis, B., Salles, J.-M., Bielsa, S., Richard, D., Martin, G., Pujol, J.-L., 2009. Approche économique de la biodiversité et des services liés aux écosystèmes. Contribution à la décision publique, Paris, La Documentation française.

Commissariat général au développement durable (CGDD), 2011. Évaluation économique des services rendus par les zones humides - complémentarité des méthodes et monétarisation. Études $\mathcal{E}$ documents, 50, http:/ / www.developpement-durable.gouv.fr/IMG/pdf/ED50.pdf.

Commissariat général au développement durable (CGDD), 2013. Avancées et enseignements pour la valorisation des services rendus par les zones humides, Le point sur, 157, http:/ /www.developpement-durable.gouv.fr/IMG/pdf/ LPS157.pdf.

Commission européenne, 1997. Premier texte de proposition de directive du conseil instituant un cadre pour l'action communautaire dans le domaine de l'eau. $\operatorname{COM}(97) 49$ Final - 97/0067(syn), Bruxelles, 26 février 1997.

De Abreu, B., 2001. La négociation de la directive-cadre sur l'eau. Place et rôle des références économiques dans le processus, Mémoire de DEA, Université de Paris-X-Nanterre, Nanterre.

De Groot, R., Blignaut, J., van der Ploeg, S., Aronson, J., Farley, J., Elmqvist, T., 2013. Benefits of investing in ecosystem restoration, Conservation Biology, 27, 1286-1293.

Garambois, N., Devienne, S., 2010. Évaluation de systèmes de production innovants inscrits en agriculture durable. Le cas des systèmes bovins herbagers du haut-bocage poitevin. Communication au symposium Innovation and sustainable development in agriculture and food (ISDA), Montpellier, 2830 juin, http://hal.archives-ouvertes.fr/docs/00/52/21/03/ PDF/Garambois_evaluation_de_systemes_innovants.pdf.

Heal, G., 2000. Nature and the marketplace. Capturing the value of ecosystem services, Washington (D.C.), Island Press.

Kirat, T., 2012, Économie du droit, Paris, La Découverte.

Large, A., 2008. Justification des dérogations économiques à l'atteinte du bon état des eaux en Seine-Normandie. Approches à différentes échelles. Mémoire de DEA, SupAgro, Montpellier.

Laurans, Y., 2006. La place de l'économie dans le dispositif de la Directive-Cadre, La Houille Blanche, 4, 74-80.

Laurans, Y., Bouni, C., Courtecuisse, A., Dubien, I., Johannes, B., 2001. L'évaluation économique de la théorie à la pratique : 
l'expérience des SDAGE en France, Natures Sciences Sociétés, 9, 2, 17-28.

Laurans, Y., Rankovic, A., Billé, R., Pirard, R., Mermet, L., 2013. Use of ecosystem services economic valuation for decision making: Questioning a literature blindspot, Journal of Environmental Management, 119, 208-219.

Levrel, H., Hay, J., Bas, A., Gastineau, G., Pioch, S., 2012. Coût d'opportunité versus coût du maintien des potentialités écologiques : deux indicateurs économiques pour mesurer les coûts de l'érosion de la biodiversité, Natures Sciences Sociétés, 20, 1, 16-29.

Matthei $\beta$, V., De Paoli, G., Strosser, P., 2012. Comparative study of pressures and measures in the major river basin management plans in the EU. Task $4 \mathrm{~b}$ - Costs \& benefits of WFD implementation: Final Report.

Milanesi, J., 2011. Une histoire de la méthode d'évaluation contingente, Genèses, 84, 6-24.

Ministère de l'Écologie et du Développement durable (MEDD), 2005. Évaluer les bénéfices environnementaux sur les masses d'eau. Document de travail : études - méthodes - synthèses, $\mathrm{n}^{\circ} 05-\mathrm{E} 08$.

Muller, P., 2000. L'analyse cognitive des politiques publiques. Vers une sociologie politique de l'action publique, Revue Française de Science Politique, 50, 2, 189-208.

Pearce, D.W., Seccombe-Hett, T., 2000. Economic valuation and environmental decision-making in Europe, Environmental Science E Technology, 34, 1419-1425.

Pearce, D.W., Atkinson, G., Mourato, S., 2006. Analyse coûts-bénéfices et environnement. Développements récents, Paris, OCDE.

Salles, J.-M., 2010. Évaluer la biodiversité et les services écosystémiques. Pourquoi, comment et avec quels résultats?, Natures Sciences Sociétés, 18, 4, 414-423.

Salvetti, M., 2013. Les évaluations économiques en appui à la gestion de l'eau et des milieux aquatiques, Vincennes, Onema.

TEEB (The Economics of Ecosystems and Biodiversity), 2010. L'économie des écosystèmes et de la biodiversité. Intégration de l'économie de la nature. Une synthèse de l'approche, des conclusions et des recommandations de la TEEB, http:/ / www.teebweb.org/

Reçu le $1^{\text {er }}$ août 2013. Accepté le 14 août 2014. 
wp-content/uploads/Study\%20and\%20Reports/Reports/ Synthesis\%20report/Synthesis\%20report_French.pdf.

Thompson, D.B., 2002. Valuing the environment. Courts' struggles with natural resource damages, Environmental Law, 32, 1, 57-89. 